\title{
ANTIGEN PRESENTATION IN UVEITIS
}

\author{
SHIV A. PRASAD ${ }^{1}$ and DALE S. GREGERSON ${ }^{2}$ \\ Bethesda, Maryland and Minneapolis, Minnesota
}

\begin{abstract}
SUMMARY
Experimental autoimmune uveoretinitis (EAU) is not only a valuable model for human inflammatory eye diseases, it is also a useful system for studying many aspects of immunobiology. One such aspect is self/nonself discrimination, the ability of the immune system to tolerate self molecules while responding aggressively to foreign antigens. Our laboratory has used EAU to investigate the mechanisms of $T$ cell tolerance to retinal S-antigen (S-Ag). Several mechanisms have been proposed to maintain $T$ cell tolerance to autoantigens, including clonal deletion and clonal anergy. As immunisation with S-Ag or pathogenic peptides activates uveitogenic $T$ cells, tolerance to this autoantigen cannot be due to clonal deletion. Nevertheless, tolerance acts to keep these existing autoreactive $T$ cells in a naive, or innocuous state. Here we suggest a novel mechanism - low-affinity occupancy of the autoantigenspecific $T$ cell receptor (TCR) by self-antigen - that may act in concert with the well-known mechanisms to maintain tolerance to S-Ag in the LEW rat. This model differs from clonal anergy in that the missing antigenpresenting cell (APC) activity is not a co-stimulatory function but a TCR co-ligand that increases the avidity of the interaction between the TCR and its peptidemajor histocompatibility complex (MHC) ligand. In the absence of this co-ligand only partial signals are generated through the TCR, leading to incomplete $T$ cell activation. This model was deduced from experiments with $\mathbf{T}$ cell lines and hybridomas specific for $\mathbf{S}$ Ag, which showed that: (1) autoreactive $T$ cells required a novel APC function, (2) this novel function was necessary to provide complete TCR engagement, and (3) activation of autoreactive $T$ cells was restricted to specific APC.
\end{abstract}

In experimental autoimmune uveoretinitis (EAU) $\mathrm{CD}^{+} \mathrm{T}$ cells mediate the destruction of the retinal

From: ${ }^{1}$ Laboratory of Viral Diseases, National Institute of Allergy and Infectious Diseases, National Institutes of Health, Bethesda, Maryland, USA; ${ }^{2}$ Department of Ophthalmology, University of Minnesota, Minneapolis, Minnesota, USA.

Correspondence to: Dale S. Gregerson, PhD, Department of Ophthalmology, Rm. 314, Lions' Research Bldg., 2001 6th St. SE, Minneapolis, MN USA. e-mail: grege001@maroon.tc.umn.edu. photoreceptor cells and surrounding tissues. Several retinal proteins can serve as targets of a uveitogenic immune response in the LEW rat, including Santigen $(\mathrm{S}-\mathrm{Ag}),{ }^{1}$ interphotoreceptor retinoid binding protein, ${ }^{2}$ rhodopsin ${ }^{3}$ and phosducin. ${ }^{4}$ Our laboratory has characterised an immunodominant, pathogenic sequence on bovine S-Ag, residues 270-289, represented by peptide bov-B1. The $\mathrm{T}$ cell response to bov-B1 has at least two components: in vitro proliferation to bov-B1 and adoptive transfer of severe EAU. ${ }^{5}$ As bov-B1-specific $\mathrm{T}$ cells transfer EAU, they must recognise the rat sequence in situ, yet they show little or no response to the corresponding peptide, rat- $\mathrm{B}$, in standard proliferation assays. This in vitro unresponsiveness suggested a mechanism of tolerance to the autoantigen, understanding of which may elucidate other mechanisms of $\mathrm{T}$ cell tolerance to self antigens.

Although S-Ag is a self protein, tolerance of it is not due to clonal deletion, as S-Ag-reactive T cells are elicited by immunisation. The immune system must therefore employ peripheral mechanisms to quiet these $\mathrm{T}$ cells. Peripheral tolerance may be a result of: (1) antigen sequestration behind anatomical barriers; (2) an immunosuppressive milieu surrounding the autoantigen; (3) antigen (Ag) presentation in the absence of co-stimulatory or accessory signals; or (4) a subthreshold Ag concentration for activation. The first two mechanisms would not explain $\mathrm{T}$ cell unresponsiveness in vitro. The roles of anergy and $\mathrm{Ag}$ concentration in tolerance to S-Ag are presented in this synopsis of results from our laboratory.

\section{THE T CELL RESPONSES TO RESIDUES 270-289 ON BOVINE S-ANTIGEN}

Residues 270-289 on bovine S-Ag, designated bovB1, represent a $\mathrm{T}$ cell immunodominant site. $^{5}$ Although this sequence differs by two amino acids from the rat autoantigen, bov-B1 induces a strong uveitogenic response. T cells raised to bov-B1 also adoptively transfer EAU, indicating that these T cells recognise the naturally processed rat peptide in situ. 


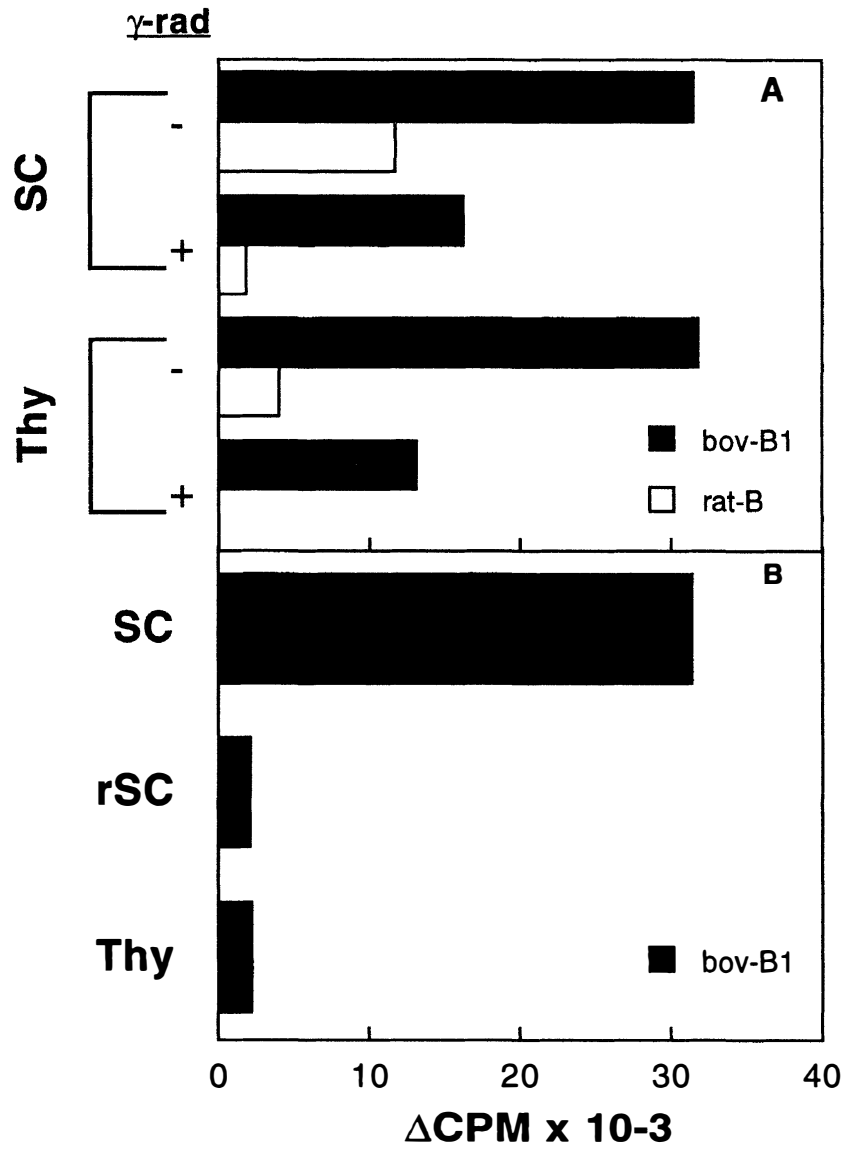

Fig. 1. Responses of the R858 T cell line and hybridoma 1C3.4. (A) R858 T cell line proliferation was stimulated with peptide bov-B1 or rat-B and irradiated (+) or nonirradiated (-) APC. (B) Proliferation of the IL-2 dependent cell line, CTLL-2, to culture supernatants from bov-B1 stimulated hybridoma 1C3.4. SC, splenocytes; $r S C$, irradiated SC; Thy, thymic APC.

Although both bov-B1 and rat-B induce active EAU, the in vitro $\mathrm{T}$ cell responses to these peptides are noticeably different.

The R858 T cell line, raised to bov-B1, proliferated in vitro when bov-B1 was presented by both irradiated and non-irradiated antigen-presenting cells (APC) (Fig. 1A). Although R858 adoptively transferred EAU, thereby recognising the naturally processed fragment of rat $\mathrm{S}-\mathrm{Ag}$, it proliferated to rat$\mathrm{B}$ in vitro only with non-irradiated APC. These data suggested that gamma $(\gamma)$-radiation had an inhibitory effect on optimal Ag presentation. As APC irradiation is a routine part of $\mathrm{T}$ cell proliferation assays, it is highly unlikely that it impaired normal APC functions such as antigen processing, MHC expression and co-stimulation. Indeed, as will be shown in subsequent experiments, the radiosensitive APC activity was not consistent with any of these processes. To characterise this radiosensitive activity, we used $\mathrm{T}$ cell hybridomas as a homologous responder population.

Although T cell hybridomas for the R858 T cell line responded to both bov-B1 and rat-B, both responses were sensitive to APC irradiation. Nevertheless, the phenotype in question, requirement for a radiosensitive APC activity, was preserved (Fig. 1B).

The inability of irradiated splenocytes to stimulate hybridoma interleukin-2 (IL-2) secretion indicated a defect in $\mathrm{Ag}$ presentation. These APC still expressed functional peptide-MHC complexes which occupied the $\mathrm{T}$ cell receptor (TCR). This was demonstrated with an assay for activation-induced cell death (AICD), a consequence of TCR occupancy. ${ }^{6}$ This assay showed that irradiated APC did indeed present peptide-MHC complexes to the hybridoma TCR (Fig. $2 A$ ), although only non-irradiated splenocytes stimulated IL-2 production (Fig. $2 B$ ). Furthermore, irradiated splenocytes stimulated AICD as efficiently as did non-irradiated splenocytes over a wide $\mathrm{Ag}$ titration. ${ }^{7}$ Since irradiated splenocytes presented bov-B1 for TCR occupancy as efficiently as nonirradiated splenocytes, it is improbable that peptide trimming, peptide-MHC association and MHC transport are the radiosensitive APC activities in question. ${ }^{8}$ Another possibility, that irradiated APC
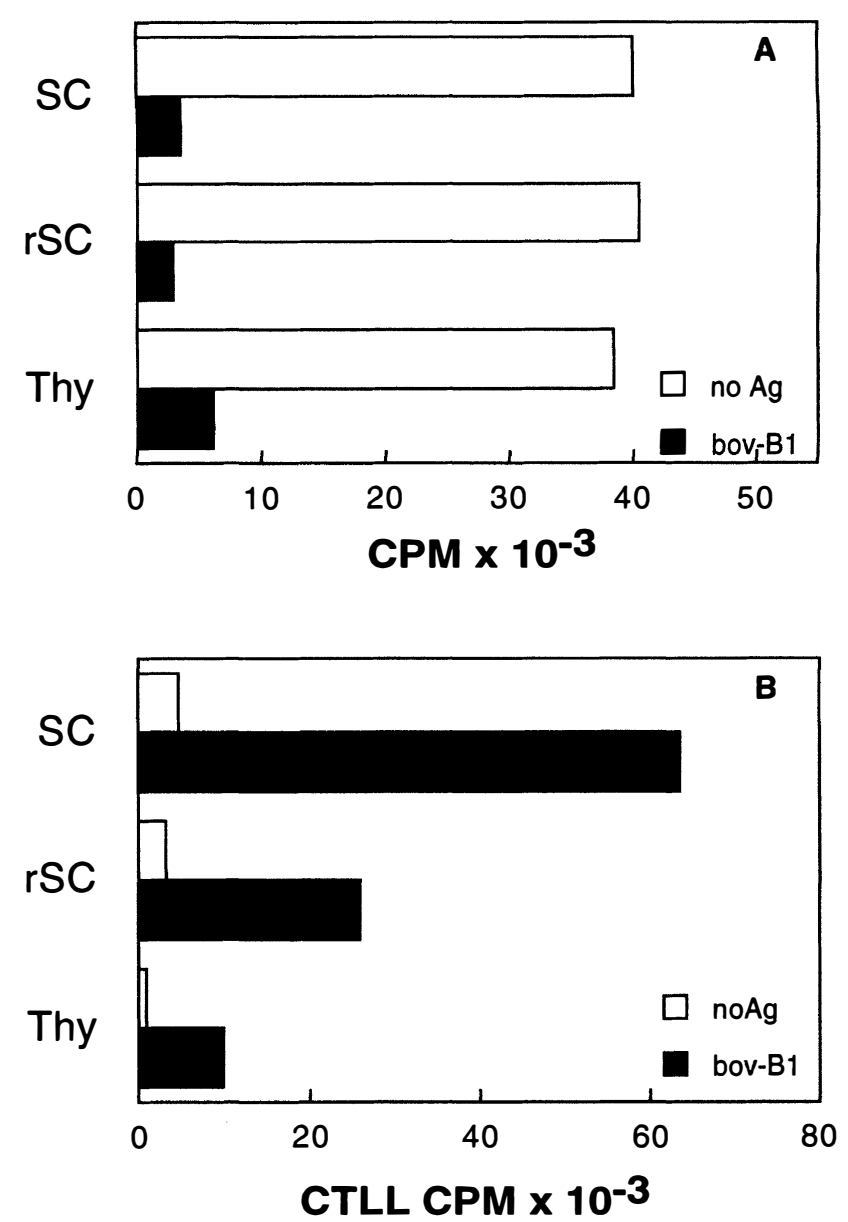

Fig. 2. Dissociation of $A I C D$ and $I L-2$ secretion. $A g$ presentation by both non-irradiated and irradiated splenocytes induced growth arrest $(A)$ in hybridoma 1C3.4, whereas only non-irradiated splenocytes stimulated $I L-2$ secretion (B). CTLL, cytotoxic T lymphocyte line. 

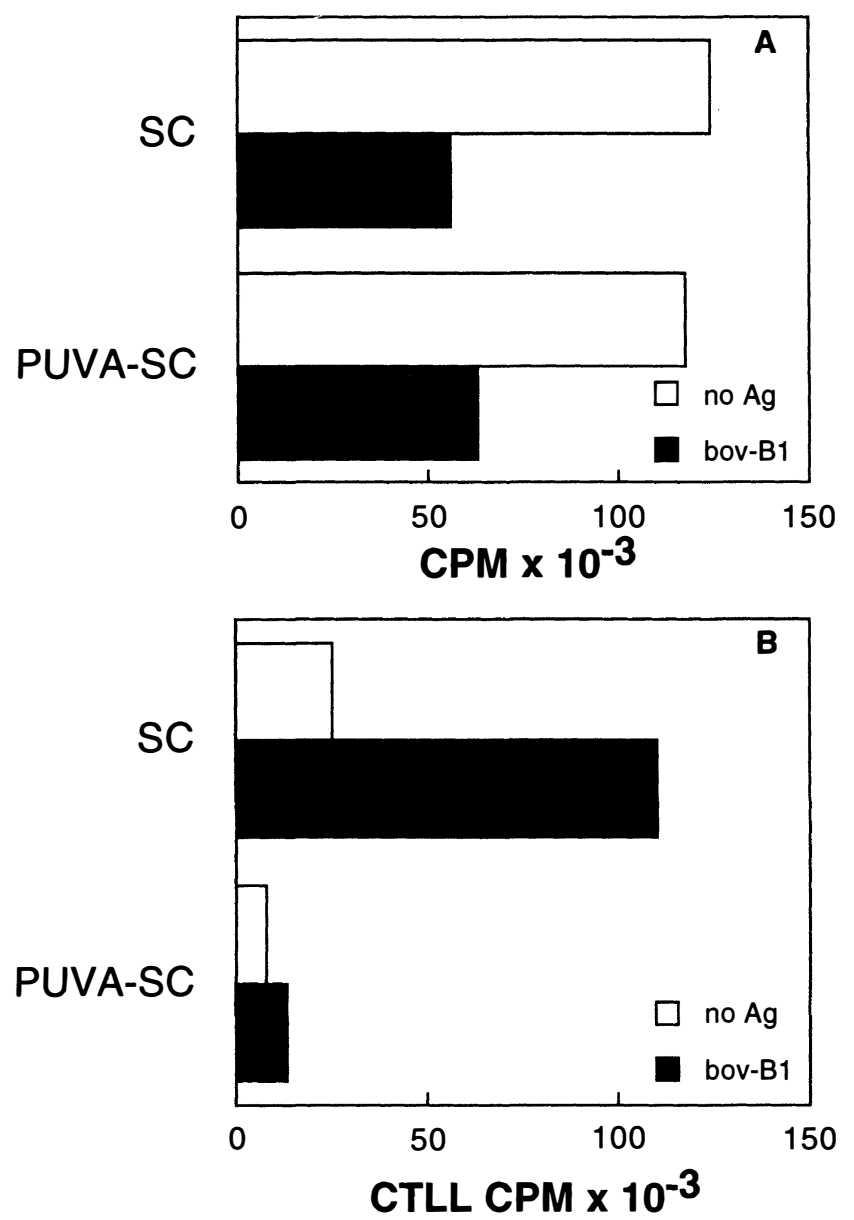

Fig. 3. Inhibition of APC macromolecular synthesis. $P U V A$-treated splenocytes sufficiently presented bov-B1 for $\operatorname{AICD}(A)$, but were unable to stimulate IL-2 secretion (B).

suppressed hybridoma IL-2 secretion, was excluded by showing that hybridoma IL-2 secretion in response to non-irradiated APC was not compromised when irradiated APC were included in the cultures. $^{7}$

\section{TARGET OF GAMMA-RADIATION}

An unusually low dose of $\gamma$-radiation, as little as $200 \mathrm{R}$, abrogated this APC activity. ${ }^{7}$ This suggested that the target of $\gamma$-radiation may be transcription of a gene whose product is not expressed on resting APC and must be upregulated after contact with the $\mathrm{T}$ cell hybridoma. This hypothesis predicts that inhibition of APC macromolecular synthesis would have the same outcome as $\gamma$-radiation. Different inhibitors of transcription were used to explore this possibility.

Psoralens are low-affinity DNA intercalators which, when crosslinked by UVA irradiation (PUVA), prevent transcription. ${ }^{9}$ We took advantage of this inhibitor to ask whether APC macromolecular synthesis was required for hybridoma IL-2 secretion. Whereas neither psolarens nor UVA radiation alone affect the ability of splenocytes to stimulate IL-2

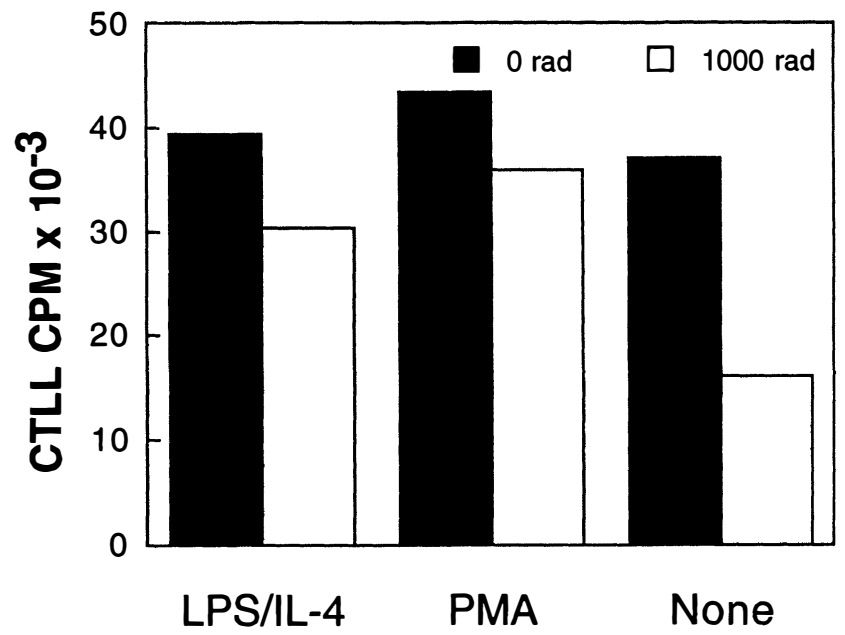

\section{APC Treatment}

Fig. 4. Radioresistance of activated APC. Splenocytes activated with PMA or lipopolysaccharide (LPS) and IL-4 stimulated IL-2 production by 1 C3.4 even after $\gamma$-radiation. This suggests that the labile function in normal splenocytes is upregulated after activation.

secretion, ${ }^{7}$ the combination of the two had the same effect as $\gamma$-radiation (Fig. 3). Treated APC induced antigen-specific AICD in 1C3.4, but did not stimulate IL-2 secretion. These results were consistent with an effect of radiation on transcription, and were reproduced in experiments using $\alpha$-amanitin, an inhibitor of transcription. ${ }^{7}$

These results suggested that the labile APC activity was functionally absent from the cell surface and upregulated after APC activation, perhaps by contact with the T cell. This model predicts that APC activation, prior to irradiation, would preserve the labile activity. When splenic APC were treated with either phorbol esters or the combination of lipopolysaccharide and IL-4 before irradiation, the labile APC activity was preserved (Fig. 4). The ability of phorbol 12-myristate 13-acetate (PMA) treatment to protect the radiosensitive activity in splenocytes did not correlate with an increase in MHC class II expression (unpublished observations). Also, supernatants from PMA-activated splenocytes did not enable irradiated splenocytes to stimulate hybridoma IL-2 production, arguing that a secreted factor was not responsible for this activity. These results are consistent with an effect of $\gamma$-radiation on the upregulation of the labile APC activity, and argue against a denaturing effect on MHC-peptide conformation.

\section{CO-STIMULATION}

As irradiated splenocytes presented Ag for TCR occupancy without IL-2 secretion, it is possible that $\gamma$-radiation inhibits a co-stimulatory function. Experiments in experimental autoimmune encephalitis have 


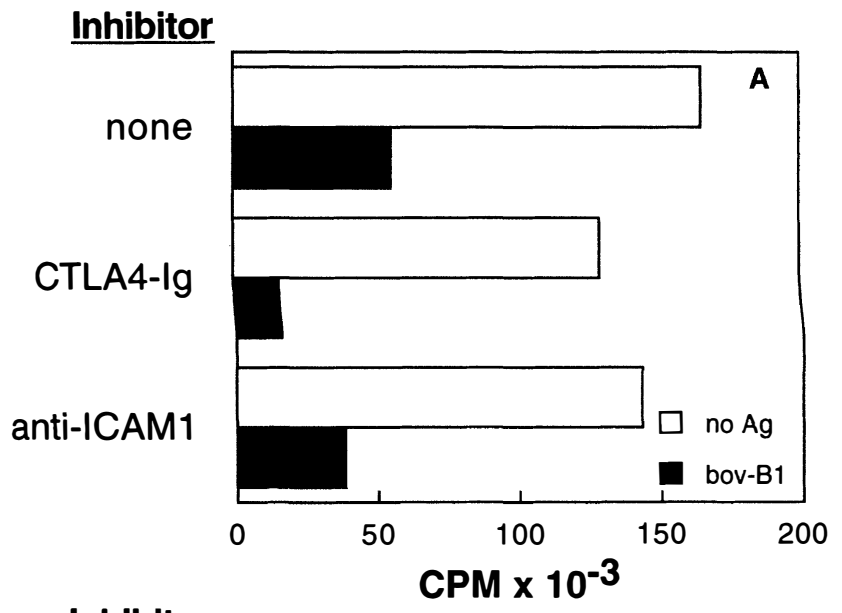

\section{Inhibitor}

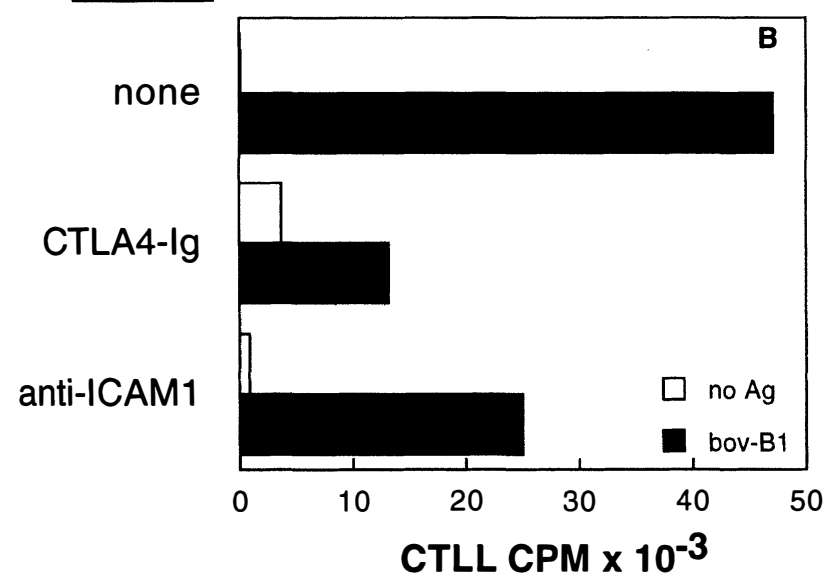

Fig. 5. Inhibition of co-stimulatory/adhesion functions. Blocking the CD28-B7 co-stimulatory interaction with CTLA4-Ig or the ICAM1-LFA1 inte action with antiICAM1 does not inhibit TCR occupancy (A), yet diminishes hybridoma IL-2 secretion $(B)$.

described a radiosensitive accessory cell co-stimulatory function, required for activation of myelin basic protein reactive $\mathrm{T}$ cell hybridomas. ${ }^{10.11}$ The bestcharacterised co-stimulatory interaction is between $\mathrm{CD} 28$, on the T cell surface, and B7 on the APC surface. ${ }^{12}$ In the absence of this second signal, TCR occupancy can result in $\mathrm{T}$ cell anergy, characterised by an inability of type $1 \mathrm{~T}$ helper cells to produce IL-2 for autocrine proliferation. To determine whether the CD28-B7 interaction was required for hybridoma IL2 secretion, CTLA4-Ig, which inhibits the CD28-B7 interaction, ${ }^{13}$ was added to cultures containing $1 \mathrm{C} 3.4$, bov-B1, and non-irradiated splenic APC. In the presence of CTLA4-Ig, 1C3.4 underwent AICD without IL-2 production (Fig. 5). If co-stimulation is indeed absent from irradiated splenocytes, engaging $\mathrm{CD} 28$ on the hybridoma should rescue IL-2 secretion. Despite the results with CTLA4-Ig, a B7-transfected cell line did not rescue IL-2 secretion by 1C3.4 stimulated with bov-B1 and irradiated splenocytes (Fig. 6). Although this showed B7 to be an unlikely candidate for the radiosensitive activity, the inhibi-

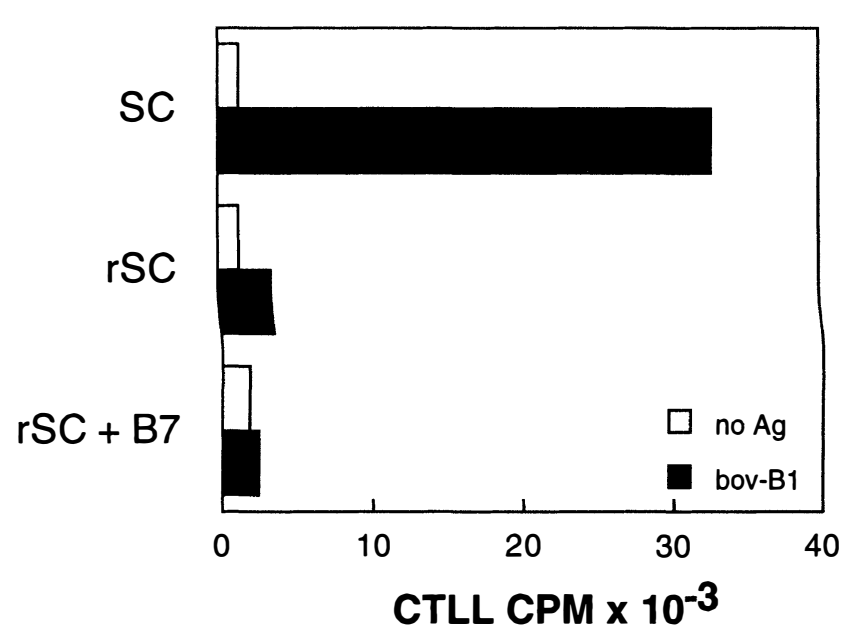

Fig. 6. IL-2 secretion is not rescued by co-stimulation. Addition of a B7 transfectant to hybridomas stimulated with irradiated splenocytes and $\mathrm{Ag}$ does not rescue IL-2 secretion.

tion of IL-2 secretion with CTLA4-Ig still suggested that the CD28-B7 interaction was involved in $\mathrm{Ag}$ presentation. As the $\mathrm{CD} 28-\mathrm{B} 7$ interaction also promotes cell-cell conjugates ${ }^{14}$ inhibition of strong intercellular adhesion by CTLA4-Ig could prevent IL-2 secretion. This interpretation is consistent with results showing that a monoclonal anti-ICAM antibody prevented IL-2 secretion with no effect on AICD (Fig. 5). The conclusion that B7 is not the radiosensitive activity is further upheld by experiments showing that B7 expression on splenocytes was not radiosensitive. ${ }^{7}$ Indeed, Ashwell et al. ${ }^{15}$ have shown co-stimulation by mouse splenocytes to be resistant at $\gamma$-radiation doses of $3000 \mathrm{R}$. Furthermore, as anti-TCR antibody alone stimulated IL-2 secretion by IC $3.4,{ }^{7}$ co-stimulation is not mandatory for IL-2 secretion.

\section{TISSUE SPECIFICITY OF ANTIGEN PRESENTATION: DO B CELLS ACTIVATE AUTOREACTIVE T CELLS?}

An early observation with the R858 T cell line, and later the R858-derived $\mathrm{T}$ cell hybridomas, was the tissue specificity of $\mathrm{Ag}$ presentation. Whereas both splenic and thymic APC induced R858 proliferation to bov-B1, only splenic APC induced rat-B-specific proliferation (Fig. 1A). As $\mathrm{R} 858$ responded to rat-B, presented by a mixture of splenic and thymic APC, it is unlikely that thymic APC exert a suppressor activity in these experiments. This tissue specificity was also seen with the hybridoma 1C3.4, which secreted IL-2 again in response to splenic, but not thymic APC (Figs. $1 B, 2 B$ ). Although thymic APC express low levels of MHC class II, they induce AICD as efficiently as splenic APC (Fig. 2A), arguing that class II expression on thymic APC was not a limiting factor. 


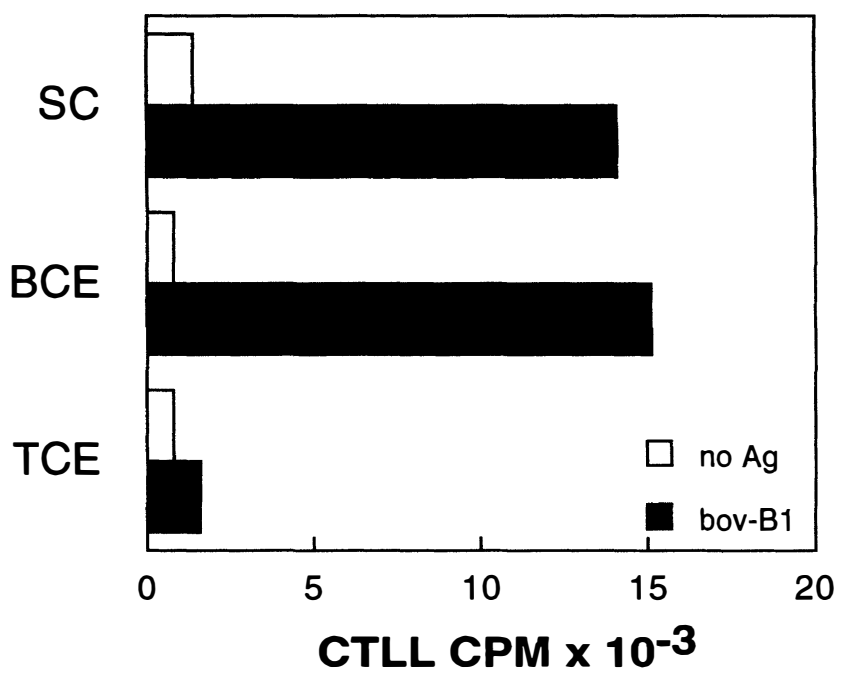

Fig. 7. Ag presentation by splenocyte fractions. Separation of splenocytes into B-cell-enriched $(B C E)$ or T-cellenriched (TCE) fractions shows that IL-2 secretion by hybridoma $1 C 3.4$ is more potently induced with the BCE fraction.

The previous result, that APC treated with PMA or LPS/IL-4 are potent APC in this system (Fig. 4), suggested that hybridoma IL-2 secretion would be promoted by the splenic APC fractions containing activated cells. When splenocytes were separated into low-density (activated) and high-density (resting) cells, the low-density cells were more potent stimulators of hybridoma IL-2 secretion. ${ }^{8}$

The differential abilities of splenic and thymic APC, and low-density and high-density splenocytes, to stimulate R858 or its hybridomas suggested that a tissue-specific APC was required for optimal Ag presentation. By examining the cells present in the various APC populations, one can make a logical inference as to the identity of this APC. The lowdensity splenocyte fraction contains activated lymphocytes, macrophages and dendritic cells, implicating one or more of these subsets as the APC required for hybridoma IL-2 secretion. However, macrophages and dendritic cells are also present in the thymus. The striking difference between splenocytes and thymocytes, and activated and resting splenocytes, is the B cell content. B cells are much more abundant in the spleen than in the thymus and are enriched by collecting activated splenocytes. This suggests that B cells were the requisite APC for stimulating IL-2 secretion by these autoreactive $\mathrm{T}$ cells.

Separation of splenocytes into T-cell-enriched and B-cell-enriched populations showed that optimal Ag presentation segregated with the latter (Fig. 7). As the enrichments were done by panning with antiTCR or anti-Ig antibodies, neither population was biased for or against macrophages or dendritic cells. Furthermore, neither adherent cells nor purified $\mathrm{T}$ cells stimulated hybridoma IL-2 production. ${ }^{8}$ These data are in agreement with the hypothesis that B cells are the necessary APC for hybridoma IL-2 secretion.

Data from other systems also point to B cells as the APC that best stimulate autoreactive T cells. Sopori et al. ${ }^{16}$ have described an autoreactive $\mathrm{T}$ cell clone activated only by $\mathrm{B}$ cells and not macrophages or dendritic cells. In these experiments splenocytes, depleted of $\mathrm{Ig}^{+}$cells, were unable to stimulate this clone. Tolerance to a self- $\mathrm{Ag}$ can be broken by priming of $\mathrm{B}$ cells, cross-reactive with a tolerated self-Ag, with their specific foreign Ag. ${ }^{17}$

\section{A UNIFYING HYPOTHESIS?}

The data have indicated that, in the absence of a tissue-specific, radiosensitive APC activity, bov-B1 specific $\mathrm{T}$ cells and hybridomas are only partially activated. The two prominent questions are: (1) What is this activity, and (2) how does it dissociate IL-2 secretion and AICD? In the following paragraphs we cite the similarities between this APC activity and known TCR ligands. From these similarities we propose a model to explain the activation of autoreactive $\mathrm{T}$ cells and hybridomas in EAU.

The effect of $\gamma$-radiation in this system is inconsistent with known functions of APC. What, then, is the radiosensitive activity? We first proposed that this activity is a TCR co-ligand, ${ }^{18}$ which bears some noteworthy resemblances to minor lymphocyte stimulatory antigens (Mls), products of mouse mammary tumour viruses (MMTV). First, B cells, which are the best source of Mls-1a presentation ${ }^{19}$ and other MMTV products, ${ }^{20}$ stimulate hybridoma IL-2 secretion better than any other splenocyte population. Second, presentation of Mls-1a is diminished by low-dose $\gamma$-radiation, ${ }^{21}$ a phenomenon also observed with these autoreactive hybridomas. Third, phorbol esters and LPS/IL-4 upregulate transcription of MMTV genes ${ }^{22,23}$ and protect the radiosensitive activity in a manner consistent with enhanced gene expression. Finally, the V $\beta 8.2$ TCR gene is highly represented in uveitogenic $\mathrm{T}$ cell lines and hybridomas. ${ }^{24,25}$ This gene is often expressed by autoreactive $\mathrm{T}$ cells in both rats and mice. ${ }^{26}$ The restricted use of TCR V $\beta$ genes in autoimmunity has led to the proposal that superantigens may activate autoreactive $\mathrm{T}$ cells in the absence of specific Ag. These $\mathrm{T}$ cells would then migrate to the sites of their specific $\mathrm{Ag}$ and induce autoimmune inflammation. In our model (Fig. 8) the TCR co-ligand is required for the high-affinity engagement of the autoreactive TCR, and $T$ cell recognition of the auto-antigen in the absence of the co-ligand would be insufficient for activation. Although our model proposes a slightly different role for a superantigen-like molecule, it does not exclude the possibility that superantigens may activate autoreactive $\mathrm{T}$ cells independent of 


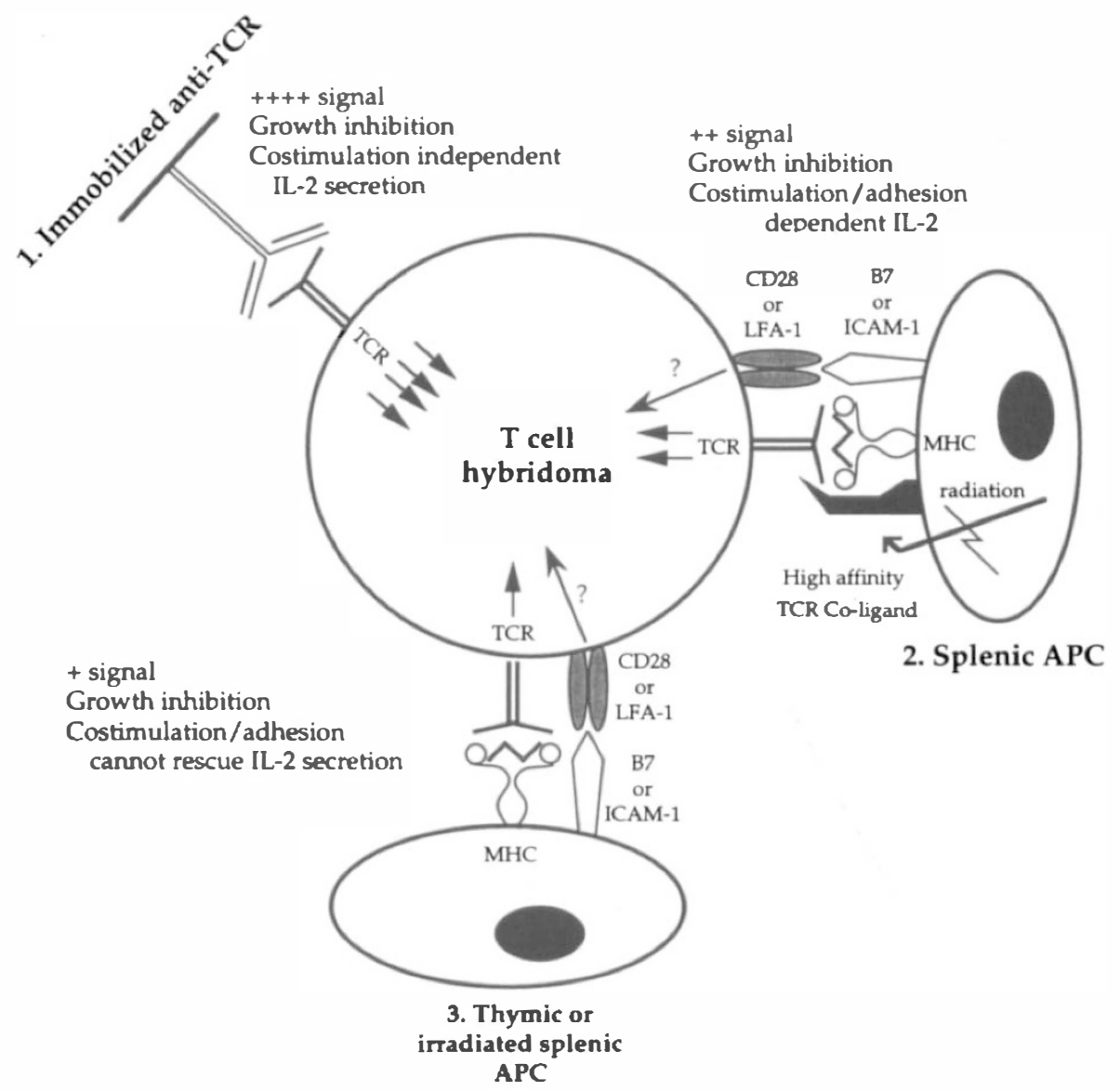

Fig. 8. Model for autoreactive $T$ cell hybridoma activation. Immobilised anti-TCR $A b$ (1) provides the strongest stimulation for the hybridomas and induces AICD and IL-2 secretion. Non-irradiated splenocytes (2) provide a lesser, but sufficient signal to induce AICD and IL-2 secretion. The latter outcome requires the TCR co-ligand, but is also dependent on the CD28-B7 and/or the LFA1-ICAM1 interactions. Thymic or irradiated splenic APC (3) provide a much weaker TCR signal in the absence of the $T C R$ co-ligand and induce only AICD.

specific Ag. How does this model then explain the dissociation of IL-2 secretion and AICD by the T cell hybridomas?

$\mathrm{T}$ cell activation was long assumed to have two separate components: TCR occupancy and costimulation. This model held that TCR occupancy with co-stimulation resulted in cytokine secretion and $\mathrm{T}$ cell proliferation, while TCR occupancy alone led to anergy with limited cytokine secretion. However, data from several laboratories have shown that TCR occupancy itself may consist of distinguishable components.

$\mathrm{T}$ cell effector functions have been separated by altering the TCR signal with partial agonists. ${ }^{27}$ These partial agonists can induce cytokine production ${ }^{28}$ or cytolysis $^{29,30}$ in the absence of $\mathrm{T}$ cell proliferation. The demonstrations that TCR signalling is not an 'all-or-nothing' event led to a model which attributes the dissociation of hybridoma IL-2 secretion and AICD to differences in the level of TCR signalling (Fig. 8). The strongest activating signal for these hybridomas is immobilised anti-TCR, which induces IL-2 secretion and AICD independent of co-stimula- tion, or other accessory-cell functions. ${ }^{7}$ Less intense, but sufficient for IL-2 secretion and AICD, is Ag presentation by non-irradiated splenic APC. Under these circumstances, APC irradiation and blocking adhesion and/or co-stimulation prevent IL-2 secretion. The lowest measurable level of TCR occupancy. occurs when $\mathrm{Ag}$ is presented by irradiated splenocytes or thymocytes, resulting in AICD without IL-2 secretion. With this low-affinity signal, IL-2 secretion is not rescued by exogenous co-stimulation.

The high activation threshold for low-affinity $\mathrm{T}$ cells may represent another level of tolerance. Immature autoreactive $\mathrm{T}$ cells, with low affinities for self-Ag, could escape negative selection in the thymus. For $\mathrm{T}$ cells specific for retinal and other neural autoantigens, the possibility of activation is greatly decreased by Ag sequestration. As activation may require not only $\mathrm{Ag}$ presentation and costimulation but a distinct co-ligand expressed by activated B cells, the likelihood that these autoreactive $T$ cells will be fully activated is even further diminished. 
Key words: Antigen presentation, Autoantigens, Immune tolerance, Retinitis, $\mathrm{T}$ lymphocytes.

\section{REFERENCES}

1. Wacker WB, Donoso LA, Kalsow CA et al. Experimental allergic uveitis: isolation, characterization and localization of a soluble uveitopathogenic antigen from bovine retina. J Immunol 1977;119:1949-58.

2. Gery I, Wiggert B, Redmond TM, et al. Uveitis and pinealitis induced by immunization with interphotoreceptor retinoid binding protein. Invest Ophthalmol Vis Sci 1986;27:1296-300.

3. Schalken JJ, Winkens $\mathrm{HJ}$, van Vugt $\mathrm{AH}$, et al. Rhodopsin-induced experimental autoimmune uveoretinitis: dose dependent clinicopathological features. Exp Eye Res 1988;47:135-45.

4. Dua HS, Lee RH, Lolley $\mathrm{RN}$, et al. Induction of experimental autoimmune uveoretinitis by the retinal photoreceptor cell protein, phosducin. Curr Eye Res 1992;11(Suppl):107-11.

5. Fling SP, Donoso LA, Gregerson DS. In vitro unresponsiveness to autologous sequences of the immunopathogenic autoantigen, S-antigen. J Immunol 1991;147:483-9.

6. Ashwell JD, Cunningham RE, Noguchi PD, et al. Cell growth cycle block of $\mathrm{T}$ cell hybridomas upon activation with antigen. J Exp Med 1987;165:173-94.

7. Prasad SA, Fling SP, Gregerson DS. A radiosensitive APC activity dissociates IL-2 secretion and activationinduced cell death by autoreactive T-cell hybridomas. Int Immunol 1995;7:1787-98.

8. Prasad SA, Fling SP, Gregerson DS. Differential APC requirements of self- and nonself-reactive $T$ cells and $T$ cell hybridomas specific for retinal S-antigen. J Autoimmun, in press.

9. Nocentini S. DNA photobinding of 7-methylpyrido[3,4-c]psoralen and 8-methoxypsoralen: effects on macromolecular synthesis, repair and survival in cultured human cells. Mutat Res 1986;161:181-92.

10. Mannie MD, Nairn R. Subset-specific co-stimulatory signals are required for IL-2 production but not growth inhibition responses by $\mathrm{T}$ cell hybrids specific for myelin basic protein. Cell Immunol 1992;140:219-36.

11. Watkins SL, Mannie MD. A unique costimulatory pathway defined with $\mathrm{T}$ cell hybridomas specific for myelin basic protein: third party costimulators restrict antigenic responses in time and space. Cell Immunol 1993;147:25-40.

12. Linsley PS, Ledbetter JA. The role of the CD28 receptor during $\mathrm{T}$ cell responses to antigen. Annu Rev Immunol 1993;11:191-212.

13. Finck BK, Linsley PS, Wofsy D. Treatment of murine lupus with CTLA4-Ig. Science 1994;265:1225-7.

14. Norton SD, Zuckerman L, Urdahl KB, et al. The CD28 ligand, B7, enhances IL-2 production by providing a costimulatory signal to T cells. J Immunol 1992;149: 1556-61.

15. Ashwell JD, Jenkins MK, Schwartz RH. Effect of gamma radiation on resting B lymphocytes. II. Functional characterization of the antigen-presentation defect. J Immunol 1988;141:2536-44.

16. Sopori M, Donaldson LA, Savage SM. T lymphocyte heterogeneity in the rat. III. Autoreactive T cells are activated by B cells. Cell Immunol 1990;128:427-37.

17. Lin R-H, Mamula MJ, Hardin JA, et al. Induction of autoreactive $\mathrm{B}$ cells allows priming of autoreactive $\mathrm{T}$ cells. J Exp Med 1991;173:1433-9.

18. Gregerson DS, Fling SP, Donoso LA, et al. Unresponsiveness to self-peptides of S-antigen in EAU: an overview of recent results. Curr Eye Res 1992;11:6774.

19. Webb SR, Okamoto A, Ron Y, et al. Restricted tissue distribution of Mls ${ }^{\mathrm{a}}$ determinants: stimulation of $\mathrm{Mls}^{\mathrm{a}}$ reactive $T$ cells by $B$ cells but not dendritic cells or macrophages. J Exp Med 1989;169:1-12.

20. Jarvis C, Germain R, Hager G, et al. Tissue-specific expression of messenger RNAs encoding endogenous viral superantigens. J Immunol 1994:152:1032-8.

21. Webb SR, Li JH, Wilson DB, et al. Capacity of small B cell-enriched populations to stimulate mixed lymphocyte reactions: marked differences between irradiated vs mitomycin C-treated stimulators. Eur J Immunol 1985;15:92-6.

22. Sharma S, King LB, Corley RB. Molecular events during $\mathrm{B}$ lymphocyte differentiation: induction of endogenous mouse mammary tumor virus proviral envelope transcripts following B cell stimulation. J Immunol 1988;141:2510-8.

23. Theunissen HJM, Paardekooper M, Maduro LJ, et al. Phorbol ester-inducible T-cell-specific expression of variant mouse mammary tumor virus long terminal repeats. J Virol 1989;63:3466-71.

24. Gregerson DS, Fling SP, Merryman CF, et al. Conserved $\mathrm{T}$ cell receptor $\mathrm{V}$ gene usage by uveitogenic T cells. Clin Immunol Immunopathol 1991;58:154-61.

25. Fling SP, Gold DP, Gregerson DS. Multiple, autoreactive TCR $V$ beta genes utilized in response to a small pathogenic peptide of an autoantigen in EAU. Cell Immunol 1992;142:275-86.

26. Heber-Katz E, Acha-Orbea H. The V-region disease hypothesis: evidence from experimental autoimmune encephalomyelitis. Immunol Today 1989;10:164-9.

27. Evavold BD, Sloan-Lancaster J, Allen PM. Tickling the TCR: selective T-cell functions stimulated by altered peptide ligands. Immunol Today 1993;14: 602-9.

28. Evavold BD, Allen PM. Separation of IL-4 production from $\mathrm{Th}$ cell proliferation by an altered $\mathrm{T}$ cell receptor ligand. Science 1991;252:1308-10.

29. Evavold BD, Sloan-Lancaster J, Hsu BL, et al. Separation of $\mathrm{T}$ helper 1 clone cytolysis from proliferation and lymphokine production using analog peptides. J Immunol 1993;150:3131-40.

30. Otten G, Germain R. Split anergy in a $\mathrm{CD}^{+} \mathrm{T}$ cell: receptor-dependent cytolysis in the absence of interleukin-2 production. Science 1991;251:1228-31. 